\title{
Kinematics Analysis of the Roller Screw Based on the Accuracy of Meshing Point Calculation
}

\author{
Yanqiang Liu, Jianshen Wang, Hongxun Cheng, and Yapeng Sun \\ School of Mechanical Engineering and Automation, Beihang University, Beijing 100191, China \\ Correspondence should be addressed to Jianshen Wang; jasenwang928@gmail.com
}

Received 31 October 2014; Revised 20 March 2015; Accepted 25 March 2015

Academic Editor: Francesco Pellicano

Copyright (C) 2015 Yanqiang Liu et al. This is an open access article distributed under the Creative Commons Attribution License, which permits unrestricted use, distribution, and reproduction in any medium, provided the original work is properly cited.

\begin{abstract}
This paper investigates the meshing behavior of the roller screw, a mechanical transmission device characterized by threaded rollers that transfer a load between the nut and the screw, by analyzing the meshing characteristics between screw and rollers. This study seeks to establish a more accurate mathematical model for the thread surface by creating a modeling process in which the max radiuses of the threads are calculated more precisely. The contact line distribution and the contact location were also calculated in order to confirm the cross section of the meshing points. In the research presented in this paper, the actual transmission ratio is analyzed and the study results in a new method to calculate the actual transmission ratio. In this study, the helical angle and the vertex angle are proven to be of great significance after a careful analysis of their influence is conducted.
\end{abstract}

\section{Introduction}

The roller screw is a mechanism used for converting rotary movement into linear displacement. Using rollers as the intermediary between the screw and the nut avoids the slide found in trapezoidal screw transmission and allows a much smaller thread than that of the ball screw, which provides the roller screw with a higher load capacity, a longer working life, higher speed, higher acceleration, and higher transmission precision. Thus, roller screws are widely used in high load, high speed, and high precision applications: in the aerospace industry, the roller screw is used for the design of landing gear [1]; in the shipbuilding industry, it is used for driving the propeller; in optical equipment, it is used for precise micro displacement [2]; it is also applied in the robotics as a kind lighter, rapid responding component [3]; and it is already applied in machine tools especially the high precision grinder.

A number of researchers have studied some aspects of the roller screw, such as its efficiency, failure mode [4], roller migration kinematics [5], contact kinematics [6], stiffness [7] capability, and limitations [8]. The roller screw is divided into several types based on the different structures [9] and the selection of structure parameters [9] is analyzed; these researches make important progress in study of roller screw; however, none of them focuses on the accurate analysis of the meshing behavior. The previous research of meshing calculation [10-12] did not consider the transmission form of the external thread meshing which would cause some unique problems for roller screw research.

This paper builds on the parametric model of the screw/nut/roller surface and the meshing between them. The meshing formulas are very complex and cannot be solved with an analytical method, so a numerical method is developed to calculate the contact line and the meshing area. Using this method, this study researches the regular changes of the contact line with different thread helical angles and vertex angles. Based on the accurate calculation of the contact line, the actual transmission ratio of the roller screw is calculated at different thread helical angles and vertex angles. Last, a brief conclusion is given about the impact this research can have on meshing performance.

\section{The Structure and the Movement Principle of the Roller Screw}

The basic structure of a planetary roller screw is shown in Figure 1. The main components include the screw, the nut, and the rollers. The screw and the nut mesh separately with 


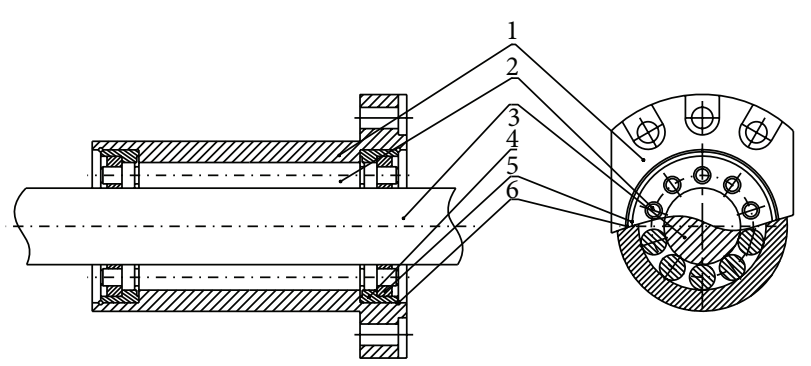
(1) Nut
(2) Roller
(4) Inner ring gear
(3) Screw
(5) Retaining plate
(6) Wire ring

FIGURE 1: Structure of planetary roller screw.

the rollers [9], which are symmetrically distributed around the screw. The gear system supports the rollers and keeps their symmetric position. The screw and the nut generally use multiple threads, while the rollers use a single thread but share the same pitch [13]. The screw and the nut always have triangular threads, but the roller can have a triangular thread or a rounded thread. When made with high machining accuracy and precise assembly, the triangular profile of the roller thread will lead to a line contact, which can provide a higher load capacity. The vertex angle of the thread is usually 90 degrees [10], but other values are also possible.

During the transmission process, the nut and the rollers need to stay static in the axial direction so that there is no slide between their thread surfaces [11]. This requires the axial displacement of the roller, because revolving along the nut thread equalizes the rotation axial displacement as shown in the following equation:

$$
\frac{\Delta \delta \cdot R_{r}^{p}}{R_{n}^{p}} \cdot \frac{p \cdot N_{n}}{2 \pi}=\frac{\Delta \delta \cdot p}{2 \pi} .
$$

$\Delta \delta$ indicates the angular displacement in the roller thread while meshing. Equation (1) can be simplified to $N_{n}=$ $R_{s}^{p} / R_{r}^{p}+2$, which is a rule that must be observed to guarantee that the roller will not move out from under the nut during the transmission process.

Assuming there is no slide between these components, the axial movement of the nut, when it is fixed circumferentially, and the screw, when it is rotated for one revolution, is defined as the transmission ratio of the roller screw. The theoretical transmission ratio of the roller screw is shown in formula (2). It can easily be seen that the transmission ratio is determined by the pitch, the lead of the screw thread, the thread directions, and the quotient of the screw radius divided by the roller radius. Thus, it is possible to achieve high speed transmission or precise positioning by choosing reasonable parameters, and it is also possible to obtain a wide range of transmission ratios:

$$
i=p_{s}+k \frac{p \cdot R_{s}^{p}}{R_{r}^{p}}
$$

In formula (2) $k$ is the roller displacement direction coefficient, 1 if the thread of the rollers takes the same helical direction as the screw, -1 if the thread of the rollers takes the opposite direction as the screw, and 0 for plane rollers.

The theoretical transmission ratio is calculated using the pitch radius based on the assumption that the meshing points lie on the cross section (the plane is determined by the screw and the roller axes). But the thread surfaces rise and twist at different degrees, which means that the meshing points between the screw and the roller actually exist mostly on only the one side of the cross section. Considering the axial free movement of the roller and the threads when crossing with each other, the actual transmission ratio should be calculated using the relative velocity as the screw and the roller conjugate across these surfaces at the meshing location.

\section{A Model of the Screw and Nut Meshing with the Roller}

3.1. A Model of the Screw, Nut, and Roller Surfaces. The surfaces of the screw, the nut, and the roller are all triangular threads, and they share the same modeling method, which means that the formulas for the thread surface modeling omit the subscripts (e.g., $R$ is used, instead of using $R_{s}, R_{n}$, or $R_{r}$ when developing the surface equation for the screw, the nut, or the roller). As is shown in Figure 2, the coordinate system $S(O, x, y, z)$ is fixed with the screw/nut/roller at the center of an end face. The coordinate system $S_{3}\left(O_{3}, x_{3}, y_{3}, z_{3}\right)$, in which the thread profile is measured, rises along the helical line with its origin $R^{p}$ from the axis, and with its $x_{3} z_{3}$ plane perpendicular to the helical line.

The transformation matrix used to convert the coordinate system $S_{3}\left(O_{3}, x_{3}, y_{3}, z_{3}\right)$ to $S(O, x, y, z)$ can be developed from Figure 2, resulting in the following matrix formula:

$$
{ }_{0}^{3} T=\left[\begin{array}{cccc}
\cos \theta & -\cos \alpha \sin \theta & \sin \alpha \sin \theta & R^{p} \cos \theta \\
\sin \theta & \cos \alpha \cos \theta & -\sin \alpha \cos \theta & R^{p} \sin \theta \\
0 & \sin \alpha & \cos \alpha & \theta R^{p} \tan \alpha \\
0 & 0 & 0 & 1
\end{array}\right],
$$

where $\alpha$ is the helical angle, which can be calculated with the following formula:

$$
\alpha=\arctan \left(\frac{N \cdot p}{2 \pi R^{p}}\right) .
$$

The vertex angle is measured from the cross section as shown in Figure 3. Point $E$ is on the thread surface; $E_{x_{3}}, E_{y_{3}}$, and $E_{z_{3}}$ are the coordinates on axes $x_{3}, y_{3}$, and $z_{3}$. The coordinate of point $E$ in the coordinate system of $S_{3}\left(O_{3}, x_{3}, y_{3}, z_{3}\right)$ is as shown in the following formula:

$$
\vec{r}_{3}=\left[\begin{array}{c}
R-R^{p} \\
\left(R^{m}-R\right) \tan \beta \sin \alpha \\
\left(R^{m}-R\right) \tan \beta \cos \alpha \\
1
\end{array}\right]
$$




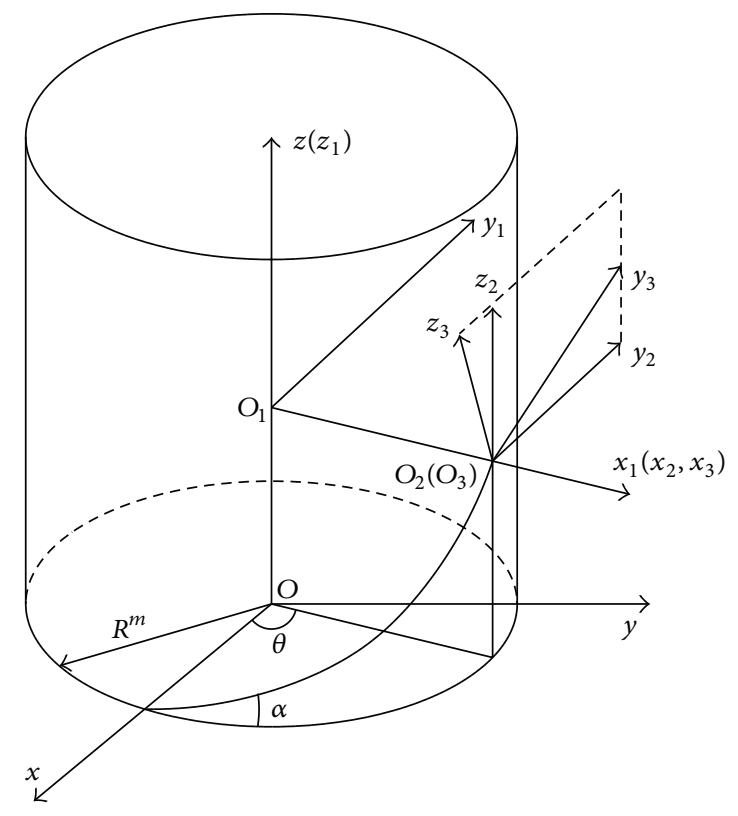

Figure 2: Thread surface coordinate system.

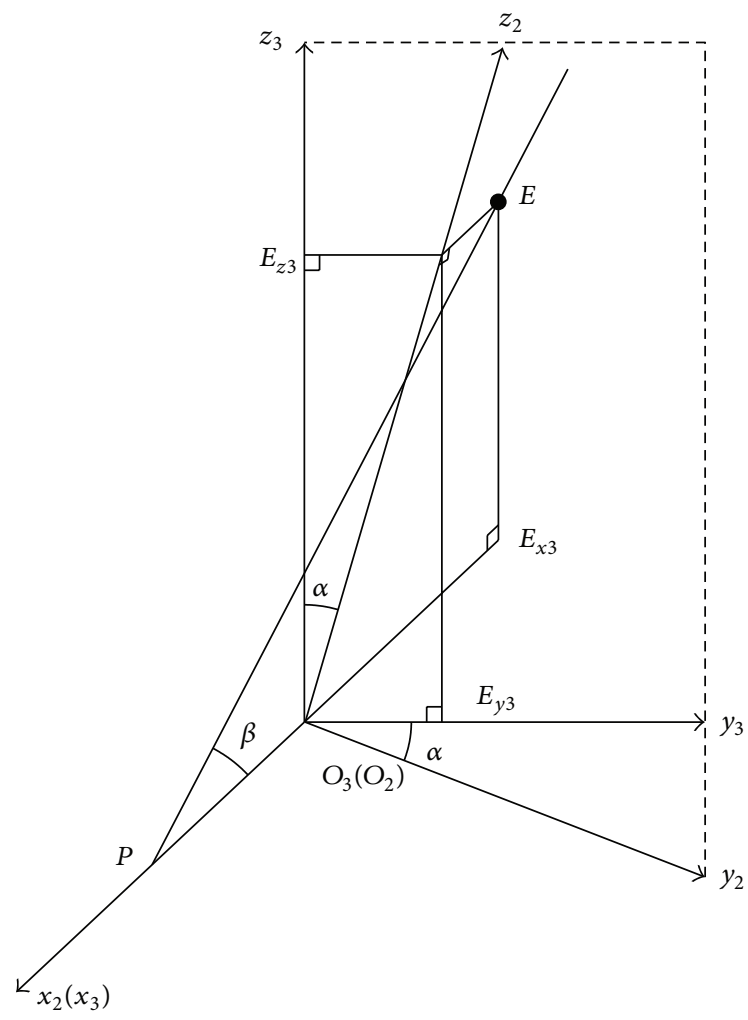

FIGURE 3: The coordinates of the meshing point. to characterize the models of the screw/nut/roller thread surface. Consider the following:

$$
\vec{r}={ }_{0}^{3} \operatorname{Tr}_{3}=\left[\begin{array}{c}
R \cos \theta \\
R \sin \theta \\
\left(R^{m}-R\right) \tan \beta+\theta R^{p} \tan \alpha \\
1
\end{array}\right] .
$$

$R^{m}$ can be calculated as shown in formula (7). $\lambda_{p}$ is the quotient of the thread tooth height divided by the pitch and $\lambda_{u}$ is the proportion of the crest part divided by the pitch radius taken from the tooth height. $\lambda_{u}$ decreases with the increase of the pitch in order to keep the max radius from becoming too large:

$$
R^{m}=R^{p}+\lambda_{p} \lambda_{u} p \cot \beta .
$$

This model can be distinguished from the ones built in the previous researches $[6,9,13]$ by highlighting the following aspects. First, we can note that the roller thread profile is a straight line instead of a circular arc, as has been assumed in previous papers. This straight line method reduces the processing difficulties found in engineering applications and could provide excellent performance with precise machining technology though curved flanks are applied to the current actual products. Second, we can note that the max radius, which impacts the thread size and the helical angle, is no longer seen as being free from the influence of alterations to the pitch. These advantages make the model more accurate, because they reflect the actual thread characteristics better.

3.2. A Model of the Meshing. The screw/nut thread surface meshes externally/internally with the roller surface; the pitch diameters are tangent; the screw axis parallels the roller axis; and the upper/lower screw surface meshes with the lower/upper roller surface [12]. The numerical assembly method calculates the surface of the roller with that of the screw/nut, thus preventing both the screw and the nut from moving and rotating, while fixing the roller axis a certain distance away from the screw/nut axis, which allows it to rotate. The distance between the axes is the pitch radius of the screw plus that of the roller. Now, observe how the meshing between the roller and the screw in the plane is perpendicular to their axes as shown in Figure 4. The transformation matrix for the roller rotation is shown in the following formula:
This coordinate can be converted to $S(O, x, y, z)$ by multiplying the transformation matrix ${ }_{0}^{3} T$ as shown in formula (6). Given the range in which $R$ and $\theta$ change, formula (6) will indicate the surface of a thread, which allows us

$$
{ }_{0}^{r} T=\left[\begin{array}{cccc}
\cos \varphi & -\sin \varphi & 0 & R_{s}^{p}+R_{r}^{p} \\
\sin \varphi & \cos \varphi & 0 & 0 \\
0 & 0 & 1 & 0 \\
0 & 0 & 0 & 1
\end{array}\right]
$$




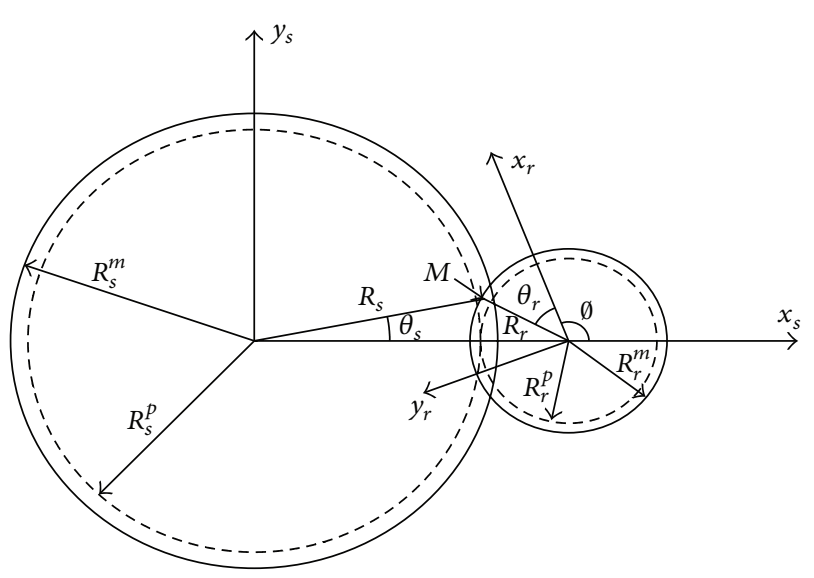

FIGURE 4: Meshing point measured in the plane perpendicular to the axis.

The roller surface as measured in the fixed coordinate system can be calculated as follows:

$\vec{r}_{r}={ }_{0}^{r} T \vec{r}_{0}=\left[\begin{array}{c}R_{r} \cos \theta_{r} \cos \varphi-R_{r} \sin \theta_{r} \sin \varphi+R_{s}^{p}+R_{r}^{p} \\ R_{r} \cos \theta_{r} \sin \varphi+R_{r} \sin \theta_{r} \cos \varphi \\ R_{r} \tan \beta_{r}-R_{r}^{m} \tan \beta_{r}+\left(\theta_{r}+\varphi\right) R_{r}^{p} \tan \alpha_{r} \\ 1\end{array}\right]$.

Setting the screw coordinate system $S_{s}\left(O_{s}, x_{s}, y_{s}, z_{s}\right)$ to coincide with the fixed coordinate system along the screw surface can now be calculated:

$$
\vec{r}_{s}=\left[\begin{array}{c}
R_{s} \cos \theta_{s} \\
R_{s} \sin \theta_{s} \\
\left(R_{s}^{m}-R_{s}\right) \tan \beta_{s}+\theta_{s} R_{s}^{p} \tan \alpha_{s} \\
1
\end{array}\right]
$$

Similarly, the nut surface is calculated as follows:

$$
\vec{r}_{n}=\left[\begin{array}{c}
R_{n} \cos \theta_{n} \\
R_{n} \sin \theta_{n} \\
\left(R_{n}^{m}-R_{n}\right) \tan \beta_{n} \cos \alpha_{n}+\theta_{n} R_{n}^{p} \tan \alpha_{n} \\
1
\end{array}\right] .
$$

The two surfaces will mesh with each other if a point where their position vectors are equal exists when the normal vectors are in the opposite direction. That point is the meshing point. According to this principle, the system of equations for calculating the meshing point between the screw and the roller can be listed [14]:

$$
\begin{gathered}
\vec{r}_{s}=\vec{r}_{r}, \\
\vec{N}_{s}=\lambda \vec{N}_{r}, \quad(\lambda \neq 0) .
\end{gathered}
$$

The normal vectors can be solved using the position vector as follows:

$$
\begin{aligned}
\vec{N}_{s} & =\frac{\partial \vec{r}_{s}}{\partial R_{s}} \times \frac{\partial \vec{r}_{s}}{\partial \theta_{s}}=\left[\begin{array}{c}
R_{s} \cos \theta_{s} \tan \beta_{s}+R_{s}^{p} \sin \theta_{s} \tan \alpha_{s} \\
R_{s} \sin \theta_{s} \tan \beta_{s}-R_{s}^{p} \cos \theta_{s} \tan \alpha_{s} \\
R_{s}
\end{array}\right], \\
\vec{N}_{r} & =\frac{\partial \vec{r}_{r}}{\partial R_{r}} \times \frac{\partial \vec{r}_{r}}{\partial \theta_{r}} \\
& =\left[\begin{array}{c}
R_{r}^{p} \sin \left(\theta_{r}+\varphi\right) \tan \alpha_{r}-R_{r} \cos \left(\theta_{r}+\varphi\right) \tan \beta_{r} \\
-R_{r}^{p} \cos \left(\theta_{r}+\varphi\right) \tan \alpha_{r}-R_{r} \sin \left(\theta_{r}+\varphi\right) \tan \beta_{r} \\
R_{r}
\end{array}\right] .
\end{aligned}
$$

Based on formulas (12) and (13), the meshing equations for the meshing between the screw and the roller can be calculated as shown in the following formula:

$$
\begin{gathered}
R_{s} \cos \theta_{s}-R_{r} \cos \left(\theta_{r}+\varphi\right)-R_{s}^{p}-R_{r}^{p}=0, \\
R_{s} \sin \theta_{s}-R_{r} \sin \left(\theta_{r}+\varphi\right)=0, \\
-R_{s} \tan \beta_{s}+R_{s}^{m} \tan \beta_{s}+R_{s}^{m} \theta_{s} \tan \alpha_{s} \\
-R_{r} \tan \beta_{r}+R_{r}^{m} \tan \beta_{r}-R_{r}^{p} \theta_{r} \tan \alpha_{r}=0, \\
R_{s} R_{r} \cos \theta_{s} \tan \beta_{s}+R_{r} R_{s}^{p} \sin \theta_{s} \tan \alpha_{s} \\
+R_{s} R_{r} \cos \left(\theta_{r}+\varphi\right) \tan \beta_{r}-R_{s} R_{r}^{p} \sin \left(\theta_{r}+\varphi\right) \tan \alpha_{r}=0, \\
R_{s} R_{r} \sin \theta_{s} \tan \beta_{s}-R_{r} R_{s}^{p} \cos \theta_{s} \tan \alpha_{s} \\
+R_{s} R_{r} \sin \left(\theta_{r}+\varphi\right) \tan \beta_{r}+R_{s} R_{r}^{p} \cos \left(\theta_{r}+\varphi\right) \tan \alpha_{r}=0 .
\end{gathered}
$$

The equations of the meshing between the nut and the roller are similar to that of the meshing between the screw and the roller, as shown in the following formula:

$$
\begin{gathered}
R_{n} \cos \theta_{n}-R_{r} \cos \left(\theta_{r}+\varphi\right)-R_{s}^{p}-R_{r}^{p}=0, \\
R_{n} \sin \theta_{n}-R_{r} \sin \left(\theta_{r}+\varphi\right)=0, \\
-R_{n} \tan \beta_{n}+R_{n}^{m} \tan \beta_{n}+R_{n}^{m} \theta_{n} \tan \alpha_{n} \\
-R_{r} \tan \beta_{r}+R_{r}^{m} \tan \beta_{r}-R_{r}^{p} \theta_{r} \tan \alpha_{r}=0, \\
R_{n} R_{r} \cos \theta_{n} \tan \beta_{n}+R_{r} R_{n}^{p} \sin \theta_{n} \tan \alpha_{n} \\
+R_{n} R_{r} \cos \left(\theta_{r}+\varphi\right) \tan \beta_{r}-R_{n} R_{r}^{p} \sin \left(\theta_{r}+\varphi\right) \tan \alpha_{r}=0, \\
R_{n} R_{r} \sin \theta_{n} \tan \beta_{n}-R_{r} R_{n}^{p} \cos \theta_{n} \tan \alpha_{n} \\
+R_{n} R_{r} \sin \left(\theta_{r}+\varphi\right) \tan \beta_{r}+R_{n} R_{r}^{p} \cos \left(\theta_{r}+\varphi\right) \tan \alpha_{r}=0 .
\end{gathered}
$$

In formula (14), $R_{s}, R_{r}, \theta_{s}, \theta_{r}$, and $\varphi$ are the parameters that determine the position of the meshing point and the pose of the roller. This nonlinear equations set cannot be solved using an analytical method, but, by applying a numerical method, the solution can be calculated if given a set of parameters. 


\section{Analysis of the Meshing between Roller and Nut}

The meshing points between the nut and the roller are calculated based on the cross section, which can be confirmed by theoretical analysis [6]. A conclusion that the helical angle of the nut thread equals that of the roller thread can be drawn by applying formulas (1), (4), and (7). This means that the nut and the roller thread surface have the same circumferential inclination. The same vertex angles of the roller and the nut threads mean that the inclination of the radius direction is also equal. Thus, the point which is on the cross section and in the pitch radius shares the same normal direction on the nut and the roller thread surface. This point matches the meshing demonstrated in (15); this is the meshing point. The meshing on the cross section makes the meshing characteristics relatively simple: the contact radius is the pitch radius and the contact angle is zero. This relatively fixed contact makes the transmission between the nut and the rollers stable and easy to analyze.

So, the remaining analysis will emphasize the meshing between the screw and the rollers, which have an obvious effect on the transmission process.

\section{Analysis of the Meshing between Roller and Screw}

Unlike the nut, the screw meshes with rollers outside the cross section. If the screw and roller mesh with each other in the cross section, that will make $\theta_{s}$ equal to 0 . If $\theta_{s}=0$ is taken into formula (14), it could be solved that

$$
\begin{gathered}
R_{s}=R_{s}^{p}, \\
R_{r}=R_{r}^{p}, \\
\alpha_{s}=-\alpha_{r}, \\
\beta_{s}=-\beta_{r} .
\end{gathered}
$$

This means that, when meshing with each other, the axial displacement generated by rotation of roller equals the axial displacement along the screw thread but of different directions. So there are no axial relative movements between roller and screw and the rotary movement of screw will not be converted into linear displacement. Thus the roller and screw must mesh outside the cross section.

The core parameter for the meshing behavior between roller and screw is the helical angles which are determined by $p$ and $R^{p}$. The helical angle of screw is different from that of roller; they are mutually independent and will influence the meshing characteristics together. The vertex angle is also important parameter which affects the meshing between roller and screw.

5.1. Contact Lines. The meshing point's distribution largely determines the meshing characteristics of the roller screw, because it is the basis for the motion of the meshing point and the force of the transfer between the screw and the rollers. The solution to formula (15) gives us the contact line of the meshing surface. Though the continuous function of the contact line could not be solved, the numerical solutions of a series of the meshing point can be formed by the contact curve. According to the circumferential symmetry of the meshing, the mesh area is the helical surface generated when the contact line rotates along the axis. Figure 5(a) shows the meshing between the screw and the roller threads and Figure 5(b) shows the specific features of the meshing point. The screw and the roller threads have helical angles of different size and directions, so the helical lines cross with each other. An appropriate incline and twist make the crossing surfaces mesh with each other. The difference between the helical angles ensures that the meshing points are located on one side of the cross section. The upper/bottom surface of screw thread meshes with the bottom/upper surface of the roller thread, thus forming a contact line.

The contact line is a basic and an important piece of data for the study of meshing characteristics, because it reflects some important meshing parameters, such as the contact radius and the contact angle. It also has a close connection with the transmission ratio, the edge contact, and some of the other meshing performance measurements. The contact radius $R_{s}\left(R_{r}\right)$ and the contact angle $\theta_{s}\left(\theta_{r}\right)$ are not structure parameters, but they are parameters used to locate the meshing points; they are measured in a plane which contains the meshing point and are vertical to the axes.

Apply numerical method to calculate the locations of meshing points and to determine the contact line and the meshing area. During the calculation, the screw pitch radius is $9.75 \mathrm{~mm}$, the roller pitch radius is $3.25 \mathrm{~mm}$, and the number of screw threads is 3 . The helical angle and the vertex angle take different values and the other parameters are worked out based on the parameters already provided.

The calculation results show that the surfaces of the screw and the roller are the contact line and the meshing line is always on only one side of the cross section. In Figure 6, contact lines are calculated using the condition of the different helical angles while other parameters remain the same. $A^{\prime} A$ has a minimal helical angle of $1.2^{\circ}$, which is calculated with a $0.4 \mathrm{~mm}$ pitch and gradually increases to the maximum of $5.4^{\circ}$ when it comes to $I^{\prime} I$, with a pitch of $2 \mathrm{~mm}$; the pitch of each contact line is $0.2 \mathrm{~mm}$ larger than that of its right side. It can obviously be seen that the contact lines move along the circumferential direction and the shapes of the curves also change. On a single contact line, the meshing points near the root demonstrate more obvious position changes; this fact makes the contact line longer and also increases the incline in the direction of the radius. A longer contact line means less contact force, which may help reduce deformation, especially if it is paired with larger contact tooth thickness. Thus, the increasing helical angle will enhance the load capacity of the roller screw. Unfortunately, a longer and more inclined contact line may also increase the risk of generating interference, which means that the helical angle should be set within a certain limited range.

As is shown in Figure 7, the value of the vertex angle also influences the position and the shape of the contact line. The contact line on the left is the smallest and each line increases by $7.5^{\circ}$ until it reaches the largest contact line. The increase of 


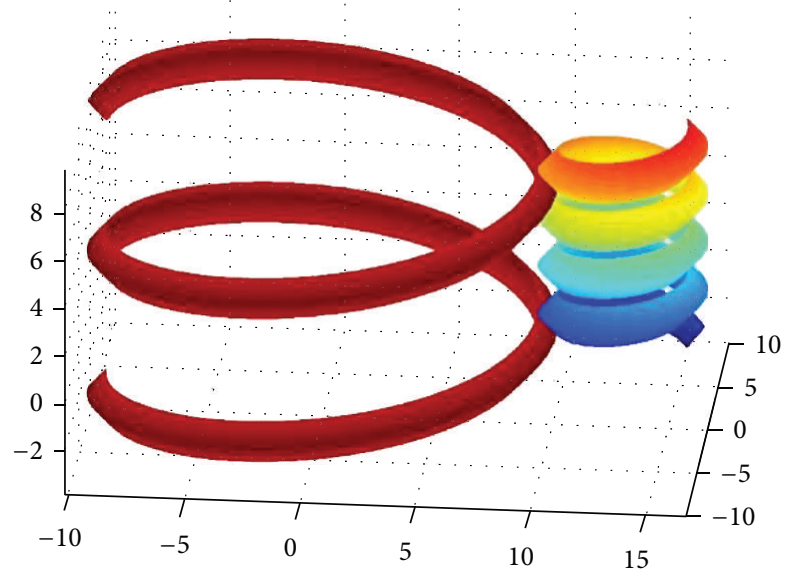

(a)

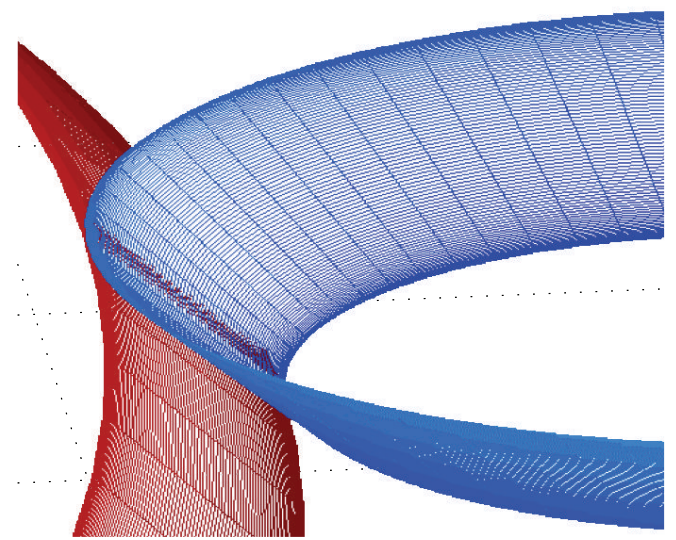

(b)

FIGURE 5: Meshing between the screw and the roller.

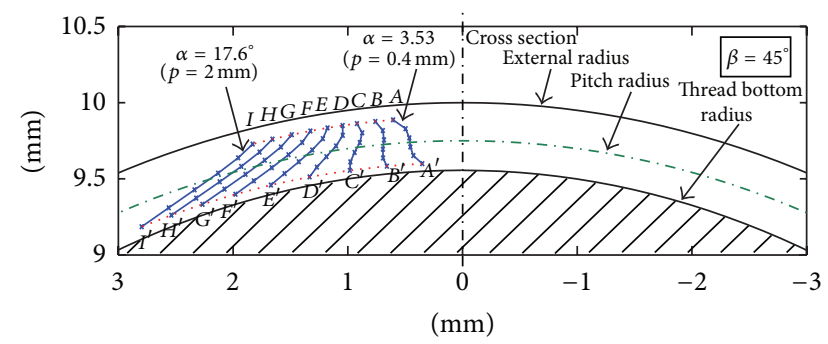

FIGURE 6: The contact lines under different helical angle conditions.

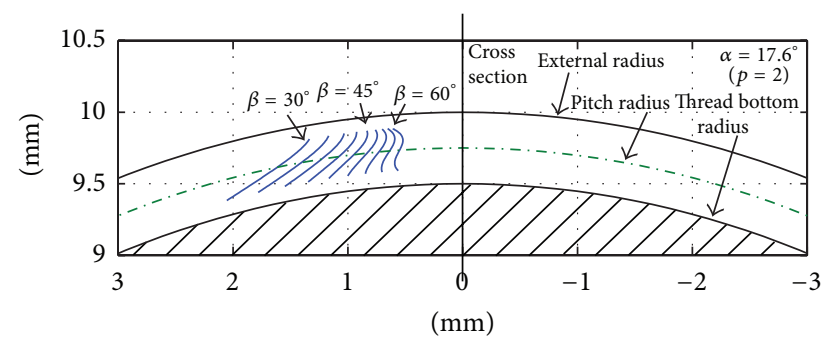

FIGURE 7: Contact lines under different vertex angle conditions.

the vertex angle moves the contact line in the cross section and makes it shorter and closer to the radial direction. At the same time, it makes it more curved, which may also cause interference.

The distribution of the contact line will, to some extent, reflect the meshing quality. For one pair of meshing threads, the meshing quality can be evaluated based on certain aspects of the contact lines. First, the distance from the contact line to the cross section largely determines the contact radius and the contact angle, which are proven to be important factors for the principle of curvature [6]. The length of the contact line directly affects the contact force. The inclination of the contact line is also of great importance, because a steeply inclined contact line will have a substantial circumferential distance, which increases the axial displacement and is more likely to result in interference.

The line contact could enhance the loading ability of the planetary roller screw and, however, may also increase the risk of interference. In this paper, a function $f_{\text {int }}(R, \theta)$ is built to indicate the interference and help evaluate the interference risk. $f_{\text {int }}(R, \theta)$ is the $z$-axis distance between the points which share the same location in $x y$ plane and exist, respectively, on screw and roller surface. It can be calculated using formulas (9) and (10):

$$
\begin{aligned}
f_{\text {int }}(R, \theta)= & -R \tan \beta_{s}+\theta R_{s}^{m} \tan \alpha_{s}-R_{r} \tan \beta_{r} \\
& -(\theta+\varphi) R_{r}^{m} \tan \alpha_{r}+R_{s}^{m} \tan \beta_{s}+R_{r}^{m} \tan \beta_{r} .
\end{aligned}
$$

In formula (17),

$$
\begin{gathered}
R_{r}=\sqrt{\left(R_{s}^{p}+R_{r}^{p}\right)^{2}+R^{2}-2 R\left(R_{s}^{p}+R_{r}^{p}\right) \cos \theta}, \\
\theta+\varphi=\pi-\arctan \left(\frac{R \sin \theta}{R_{r}}\right) .
\end{gathered}
$$

If the value of $f_{\text {int }}(R, \theta)$ is positive, it means there is no interference. $f_{\text {int }}(R, \theta)<0$ means that interference and the interference area are shown in Figure 8 of the different helical angles and Figure 9 of different vertex angles.

It can be seen from Figure 8 that the interference area is varying with different helical angles. When the thread has a smaller helical angle of $\alpha=3.4^{\circ}$, the interference will only probably take place on the very edge of the thread and could be avoided by modifying the root or addendum. Along with the increasing of helical angle, the interference area spreads to almost the whole breadth of thread which will disable the threads meshing.

It can be seen in Figure 9 that, just like the helical angle, a changing vertex angle also has influence on the interference area, and a larger vertex angle benefits avoiding interference. 


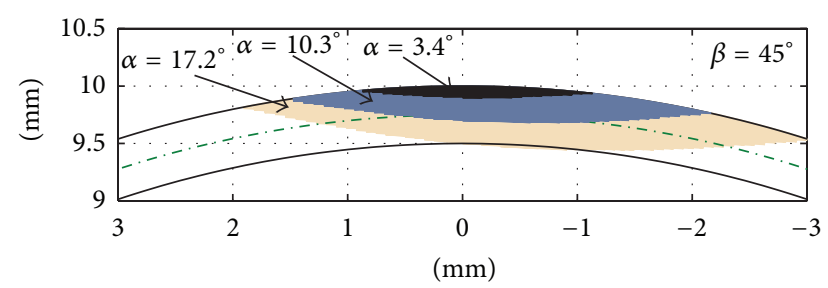

Figure 8: Meshing interference area under different helical angle conditions.

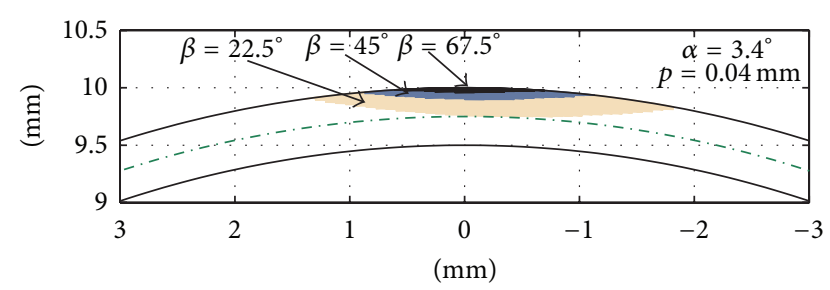

Figure 9: Meshing interference area under different vertex angle conditions.

Based on the analysis of the contact line of planetary roller screw, it can be concluded that the straight line thread roller screw can be applied in condition of small helical angle and relatively large vertex angle. Under these conditions, the line contact will increase the contact points and enhance the load capacity. With the helical angle increasing, the line contact planetary roller screw will face the risk of interference and need to be modified.

5.2. Meshing Points. In the meshing process, the contact line will create an uncertain meshing radius and is more sensitive to manufacturing and installation errors; so, the profile of the thread surface needs to be modified. The modified profile can take many shapes and will make the surface more complex, but the purpose and the principle of modifying the thread surface are the same. The modified profile must retain the indispensable contact points and avoid other contact points. The meshing point between the nut and the roller is on the cross section and in the pitch radius; these locations can guarantee a relatively static interaction between the roller and the nut, so they are chosen as the points to be retained while the other points on the contact line must be eliminated. For the surface, most areas, except the line along pitch diameters, are modified to be of a certain shape to avoid unnecessary contact, but the specific shape does not affect the meshing point calculation. So, the calculation for solving the meshing point of the modified surface can be simplified by using the helical line on the pitch diameter as the roller to solve (14). The helical line is separate from the roller thread surface, so it has the same normal vectors as is on the surface. In this way, the meshing points of the modified thread surface can be calculated.

The meshing points between the roller and the screw can still be calculated when the helical angle changes, as is shown in Figure 8. As the meshing points change from the rightmost point, which has the smallest helical angle of $1.2^{\circ}$, to the leftmost point, with the maximal helical angle of $14.6^{\circ}$, the meshing points demonstrate a trend that moves away from the pitch radius toward the thread crest, where the tooth thickness is thinner, and away from the cross section, just as the contact lines do. The meshing points would even go beyond the boundary of the thread surface if the helical angle is too large. It can be seen that the contact radius will increase if the contact angle became larger and similar regularities can be found among the meshing points with different vertex angles as is shown in Figure 9. A smaller contact angle is helpful in order to get a meshing point away from the edge of the thread, so a smaller helical angle and a larger vertex angle improve the results when the distance is taken from the thread edge in accordance with the evaluation standard. The locations of the meshing points still affect other performance factors of the roller screw, which makes the selection of parameters far more complex than this process suggests. The rest of this paper will demonstrate how the meshing point location affects the transmission ratio, which is one of the most important performance parameters of the roller screw.

\section{The Actual Transmission Ratio of an Asymmetric Meshing}

The transmission ratio is one of the basic parameters of the roller screw, which directly influences the selection of different working conditions. There exists a difference between the actual and the theoretical transmission ratio, because the meshing points are considered as if they were lying on the cross section when calculating the theoretical transmission ratio; however, it can be found that the meshing points between the screw and the roller always have a certain distance from the cross section, which indicates that the actual transmission ratio is not likely to be the same as the theoretical transmission ratio.

The actual transmission ratio can be defined as the nut axial displacement for one rotation of the screw and can be calculated using the ratio of the nut axial linear velocity and the screw angular velocity. The nut axial linear velocity equals the axial component of the screw velocity, which is indicated by $v_{z}$. The relative velocity between the roller and the screw at the meshing point can be calculated using the following formula:

$$
\vec{v}_{s r}=\left(\vec{\omega}_{r}-\vec{\omega}_{s}\right) \times \vec{r}_{s}-\vec{\omega}_{r} \times\left(R_{s}^{p} i_{s}\right)+v_{z} k_{s}
$$

The relationship of the angular velocity to the screw and the roller can be easily obtained as follows:

$$
\frac{\omega_{s}}{\omega_{r}}=\frac{2 R_{r}^{p}}{R_{s}^{p}}
$$

The relative velocity at the meshing point must satisfy the following equations:

$$
\vec{v}_{s r} \cdot \vec{N}_{s}=\vec{v}_{s r} \cdot \vec{N}_{r}=0
$$


By taking formulas (13) and (19) into (20), $v_{z}$ can be calculated as follows:

$$
\begin{aligned}
v_{z}= & \frac{R_{r}}{R_{s}} \cdot \frac{p_{s}}{2 \pi} \cdot \omega_{r} \cos \left(\theta_{r}+\varphi-\theta_{s}\right)+\frac{p_{s}}{2 \pi} \cdot \omega_{s} \\
& -\frac{R_{r}^{p}}{R_{s}} \cdot \frac{p_{s}}{2 \pi} \cdot \omega_{r} \cos \theta_{s}+R_{s}^{p} \tan \beta_{s} \omega_{r} \sin \theta_{s} .
\end{aligned}
$$

The actual transmission ratio $i_{a}$ is the quotient $v_{z}$ divided by $\omega_{s}$ :

$$
\begin{aligned}
i_{a}= & \frac{2 \pi v_{z}}{\omega_{s}}=\frac{p_{s} R_{r} R_{s}^{p}}{2 R_{s} R_{r}^{p}} \cos \left(\theta_{r}+\varphi-\theta_{s}\right)+p_{s} \\
& -\frac{p_{s} R_{s}^{p}}{2 R_{s}} \cos \theta_{s}+\frac{\pi\left(R_{s}^{p}\right)^{2}}{R_{r}^{p}} \tan \beta_{s} \sin \theta_{s}
\end{aligned}
$$

The actual transmission ratio can be calculated using the solutions to (15), as stated above, because the helical angle is an important parameter that affects transmission performance and the transmission ratio. Though $\alpha_{s}$ is not explicitly stated in formula (22), $R_{s}, R_{r}, \theta_{s}, \theta_{r}, \varphi$, and $p_{s}$ are all influenced by $\alpha_{s}$ (Figure 12).

As is shown in Figure 10, the actual transmission ratio almost proportionally increases with the growth of the helical angle, as does the theoretical transmission ratio. The rate of increase observed in the actual transmission ratio is larger, because as the meshing point deviates from the cross section it may increase the actual contact radius. It can be seen that a larger helical angle is helpful for enhancing the transmission ratio, but, as has been proven above, it will also push the meshing location to the thinner thread edge and cause interference problems, which may be harmful. So, the selection of the helical angle must take several factors into account and must satisfy the main requirements as determined by the different application situations (Figure 13).

As has been illustrated previously, the growth of the vertex angles will make the meshing point closer to the cross section. This means that there will be a smaller contact radius and that decreases the actual transmission ratio, as is shown in Figure 11. Altering the vertex angle will not change the theoretical transmission ratio, which can be seen in formula (4); however, the actual transmission ratio can be lower than the theoretical one if there is a large vertex angle. This is because the meshing points are quite close to the pitch radius when there is a large vertex angle, as is shown in Figure 9; so the screw contact radius is almost the pitch radius $\left(R_{s}=R_{s}^{p}\right)$; however, a contact angle must still exist. So, in the triangle that is composed of $R_{s}, R_{r}$, and $R_{s}^{p}+R_{r}^{p}$, the roller contact radius $R_{r}$ is larger than $R_{r}^{p}$ and $R_{s} / R_{r}$ is relatively small. This may cause a relatively smaller transmission ratio, which is why there is a lower actual transmission ratio in Figure 11.

Obviously, the actual transmission ratio is different from the theoretical transmission ratio, because the contact radius differs from the pitch radius. In most cases, the actual radius is larger, because of the deviation between the meshing point and the cross section.

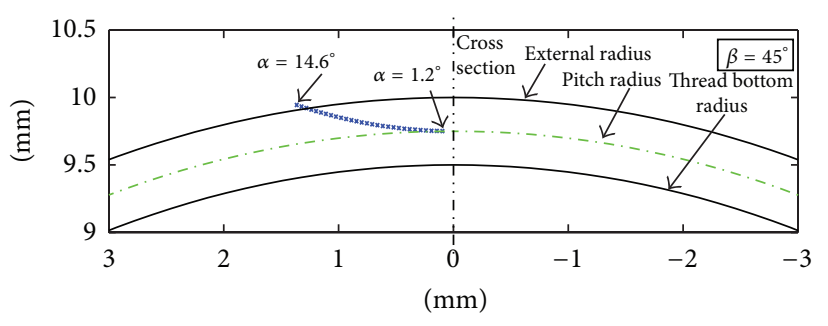

FIGURE 10: Meshing points under different helical angle conditions.

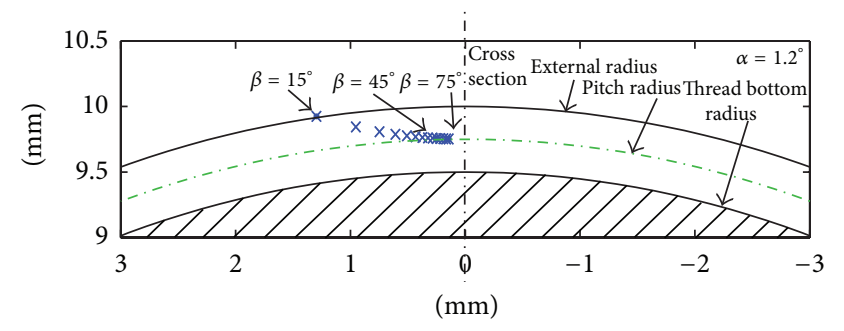

FIGURE 11: Meshing points under different vertex angle conditions.

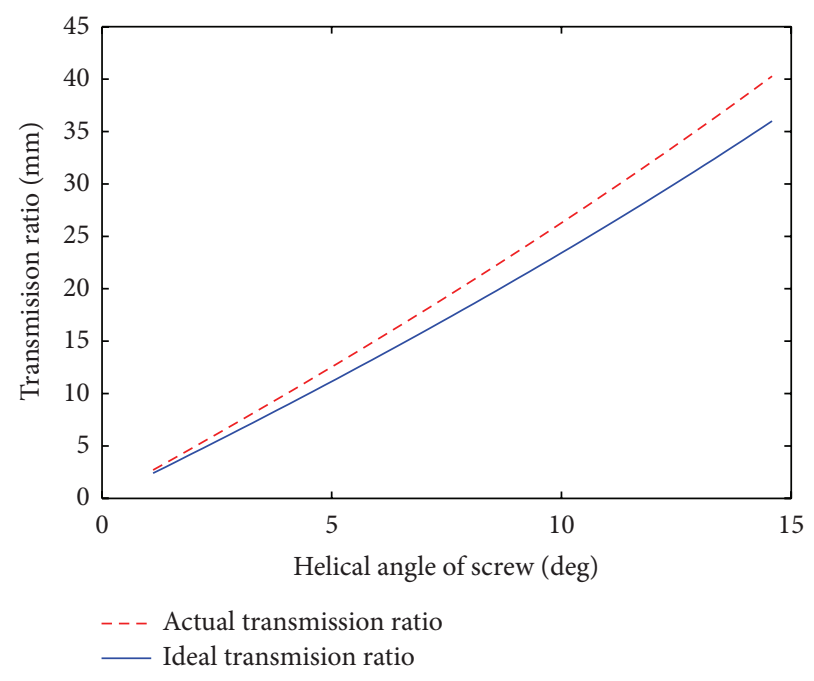

FIgURE 12: Transmission ratios under different helical angle conditions.

\section{Conclusion}

The research presented in this paper has developed a method for modeling the roller screw meshing behavior accurately and has analyzed the numerically calculated data used to evaluate the meshing characteristics. The mathematical model established herein can reflect the actual roller screw by taking several factors, which are ignored in the analytic model, into consideration due to their actual significance. As has been demonstrated, the contact lines and the meshing points can be calculated and the actual transmission ratio can be analyzed by applying a new calculation method. The helical angle and the vertex angle are proven to be of great significance for the actual meshing characteristics. They have manifested effects on the position of the meshing points and 


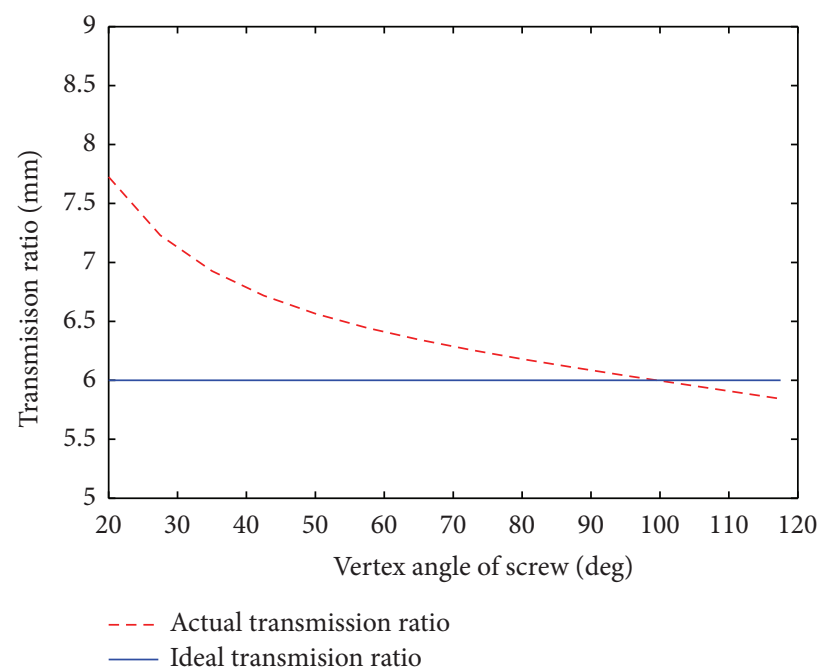

FIGURE 13: Transmission ratios under different vertex angle conditions.

will directly impact the tooth thickness and the tooth height. Based on this analysis, a larger helical angle will enhance the load capacity and the transmission ratio of the roller screw, though it may cause interference problems. A larger vertex angle will increase the load capacity significantly and helps avoid interference; however, it may increase the contact force and decrease the transmission ratio. The helical angle and the vertex angle are relatively independent parameters that can be used together to make up for the disadvantages they each cause separately. Thus, different values could be selected in order to meet the requirements of different applications and to maximize the benefits of the roller screw for different uses.

\section{Nomenclature}

\section{Given Geometric Parameters}

$R_{r}^{p}: \quad \quad$ Pitch radius of the roller $(3.25 \mathrm{~mm})$

$R_{s}^{p}: \quad$ Pitch radius of the screw $(9.75 \mathrm{~mm})$

$R_{n}^{p}$ : $\quad$ Pitch radius of the nut $(16.25 \mathrm{~mm})$

$N_{n}: \quad$ Number of threads on the nut (5)

$N_{s}: \quad \quad$ Number of threads on the screw (3)

$p: \quad$ Pitch $(0.4 \mathrm{~mm} \sim 6 \mathrm{~mm})$

$p_{s}: \quad$ Lead of screw $(1.2 \mathrm{~mm} \sim 18 \mathrm{~mm})$

$\beta_{r}, \beta_{s}, \beta_{n}$ : Half-vertex-angle of the thread of roller, screw, and nut $\left(20^{\circ} \sim 75^{\circ}\right)$

$\lambda_{p}: \quad$ Quotient of the thread tooth height divided by the pitch $(0.6 \sim 1)$

$\lambda_{u}$ : Proportion of the crest part to the tooth height $(0.35 \sim 0.65)$.

\section{Intermediate Parameters for Calculation}

$\alpha_{r}, \alpha_{s}, \alpha_{n}$ : Helical angle of roller, screw, and nut
$R^{m}: \quad$ Maximal radius (distance from the point where the upper surface of the thread meets the lower one to the axis)

$\beta_{r}, \beta_{s}, \beta_{n}$ : Half thread vertex angle of roller, screw, and nut

$\varphi$ : $\quad$ The initial position angle of the roller

$\vec{r}_{r}, \vec{r}_{s}, \vec{r}_{n}$ : Mesh point position of the vector for the roller, screw, and nut

$\vec{N}_{r}, \vec{N}_{s}, \vec{N}_{n}$ : Normal vector of the roller, screw, and nut surface at the mesh point

$R_{r}, R_{s}, R_{n}: \quad$ Distance between the mesh points to the axis of the roller, screw, and nut

$\theta: \quad$ Contact angle (the angle between $\vec{r}$ and the cross section)

$\vec{v}_{s r}: \quad$ Relative velocity between the roller and the screw

$\omega_{r}, \omega_{s}: \quad$ Angular velocity of the roller and the screw.

\section{Performance Parameters}

$i_{a}:$ Actual transmission ratio

$i$ : Theoretical transmission ratio.

\section{Conflict of Interests}

The authors declare that there is no conflict of interests regarding the publication of this paper.

\section{Acknowledgments}

This study was supported by Project 51305010 supported by National Natural Science Foundation of China and Project YCSJ-03-2013-06 supported by the Graduate Innovative Practices Foundation of Beihang University.

\section{References}

[1] W. M. Waide, "Aircraft landing gear with integrated extension, retraction, and leveling feature," U.S. Patent 7,942,366, 2011.

[2] M. S. Worthington, T. A. Beets, J. H. Beno et al., "Design and development of a high-precision, high-payload telescope dualdrive system," in Ground-Based and Airborne Telescopes III, vol. 7733 of Proceedings of SPIE, July 2010.

[3] T. Buschmann, S. Lohmeier, and H. Ulbrich, "Humanoid robot Lola: design and walking control," Journal of Physiology Paris, vol. 103, no. 3-5, pp. 141-148, 2009.

[4] P. C. Lemor, "The roller screw, an efficient and reliable mechanical component of electro-mechanical actuators," in Proceedings of the 31st Intersociety Energy Conversion Engineering Conference (IECEC '96), pp. 215-220, August 1996.

[5] M. H. Jones and S. A. Velinsky, "Kinematics of roller migration in the planetary roller screw mechanism," Journal of Mechanical Design, Transactions of the ASME, vol. 134, no. 6, Article ID 061006, 2012.

[6] M. H. Jones and S. A. Velinsky, "Contact kinematics in the roller screw mechanism," Transactions of the ASME, Journal of Mechanical Design, vol. 135, no. 5, Article ID 51003, 2013. 
[7] M. H. Jones and S. A. Velinsky, "Stiffness of the roller screw mechanism by the direct method," Mechanics Based Design of Structures and Machines, vol. 42, no. 1, pp. 17-34, 2014.

[8] Y. Hojjat and M. M. Agheli, "A comprehensive study on capabilities and limitations of roller-screw with emphasis on slip tendency," Mechanism and Machine Theory, vol. 44, no. 10, pp. 1887-1899, 2009.

[9] G. Liu and S. Ma, "New development and key technology of planetary roller screw," Journal of Mechanical Transmission, vol. 36, no. 5, pp. 103-108, 2012 (Chinese).

[10] Y. Zhao, J. Ni, and L. Lv, "Meshing calculation of roller screw," Journal of Machine Design, vol. 3, no. 20, pp. 34-36, 2003 (Chinese).

[11] R. Su, "Principle analysis of roller screw," Journal of Sichuan Institute of Technology, vol. 17, no. 3, pp. 33-36, 1998 (Chinese).

[12] Y. Q. Liu and J. S. Wang, "Simulation of crossing threaded planetary roller screw engagement," Advanced Materials Research, vol. 889, pp. 518-526, 2014.

[13] Z. Wei and J. Yang, "Optimized analysis on structural parameter for planetary roller screw," Journal of Mechanical Transmission, vol. 35 , no. 6 , pp. 44-47, 2011 (Chinese).

[14] F. L. Litvin and A. Fuentes, Gear Geometry and Applied Theory, Cambridge University Press, Cambridge, UK, 2004. 


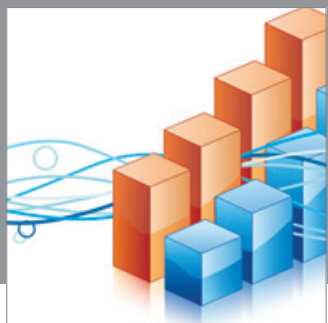

Advances in

Operations Research

mansans

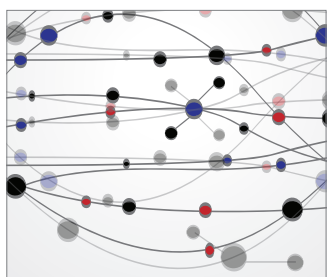

The Scientific World Journal
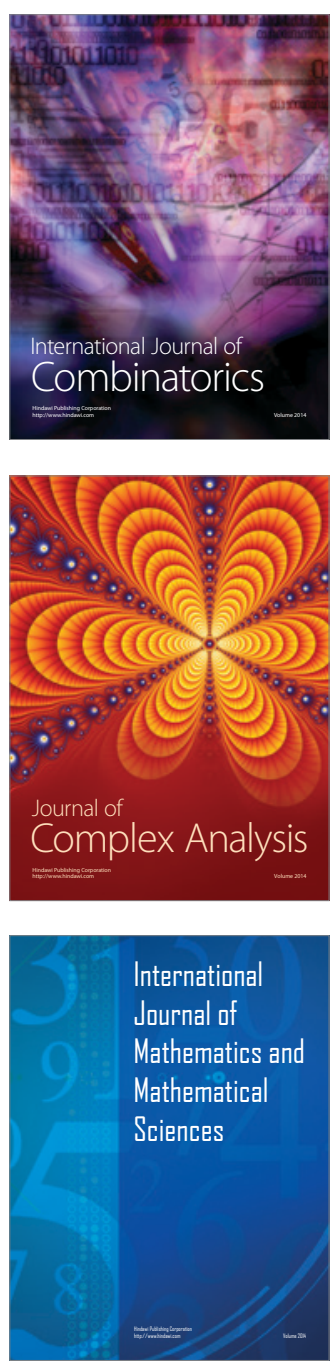
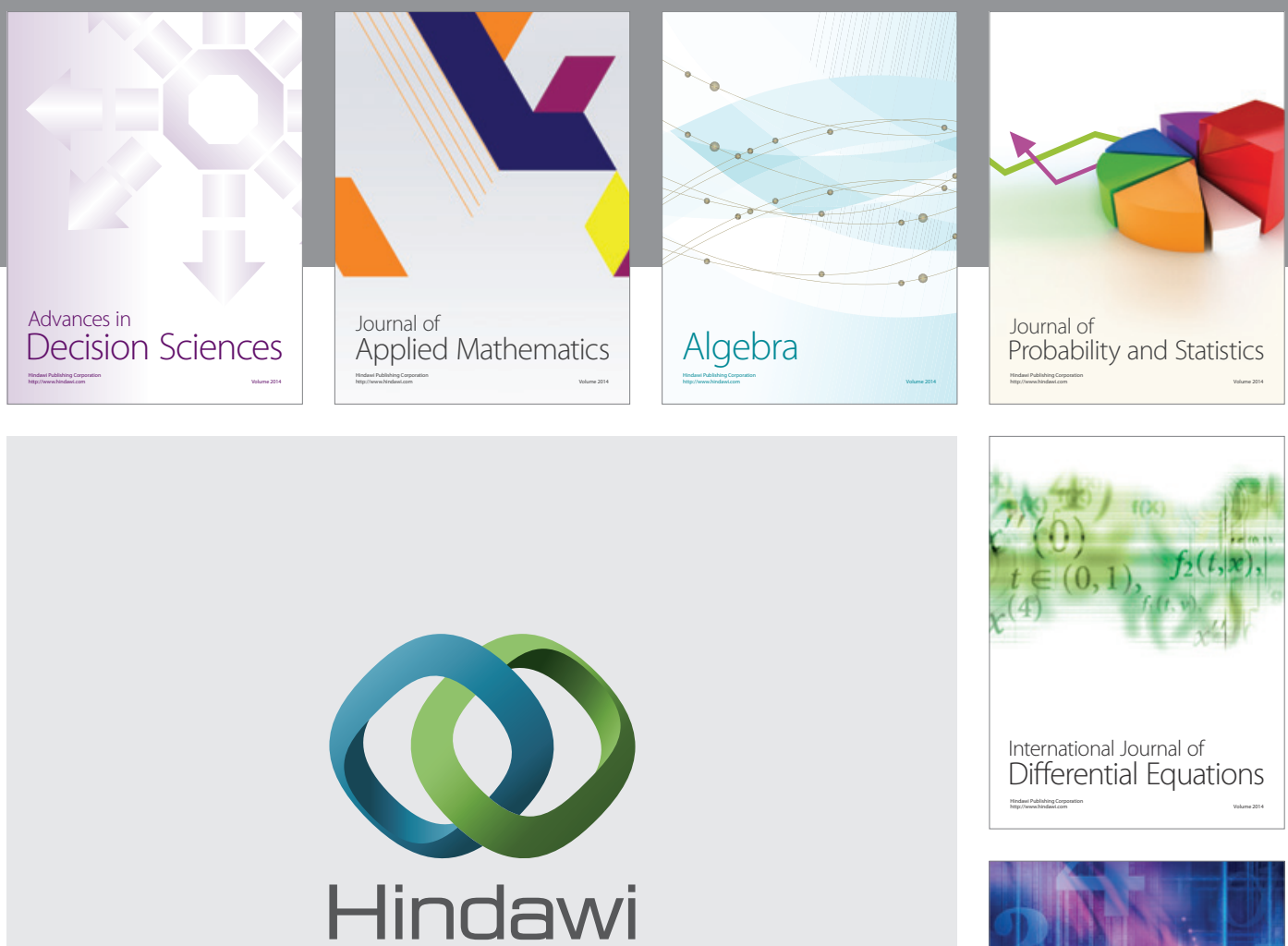

Submit your manuscripts at http://www.hindawi.com
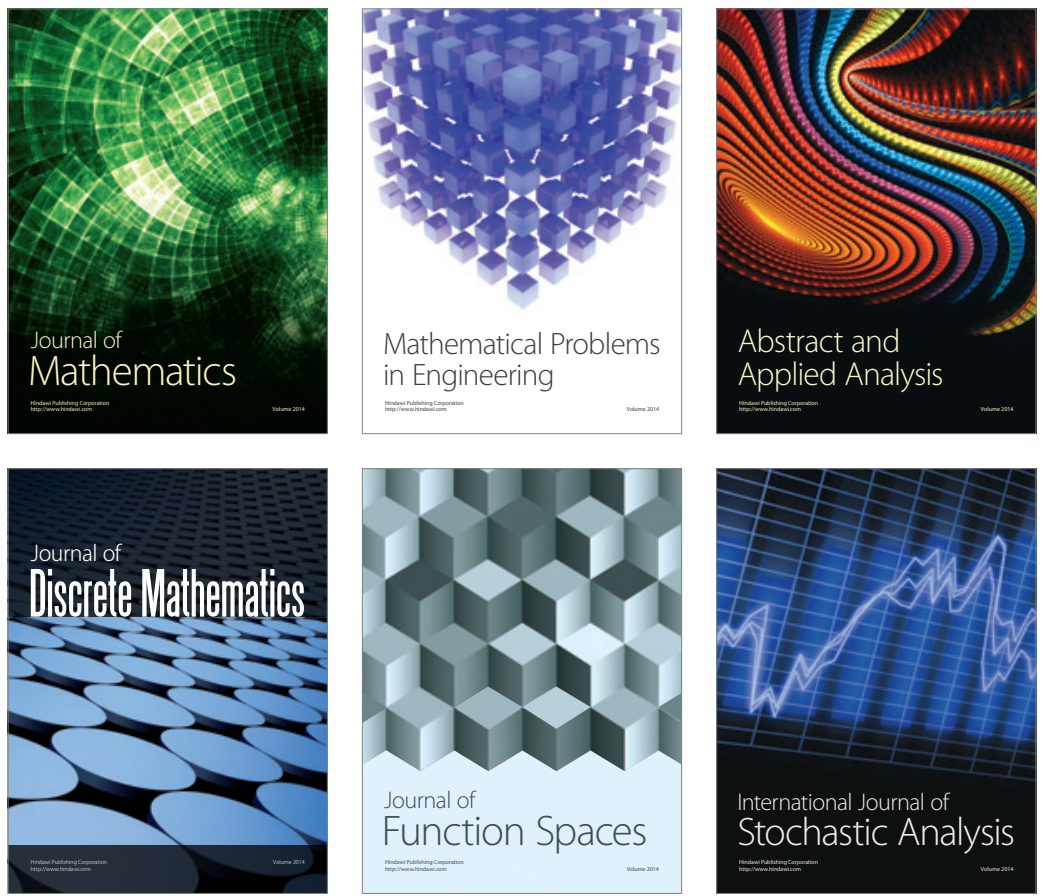

Journal of

Function Spaces

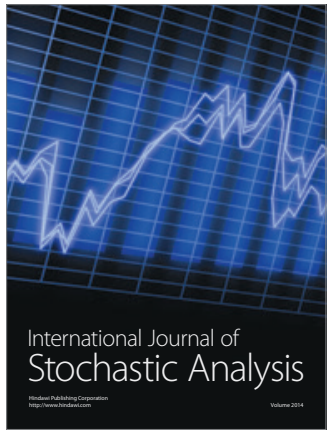

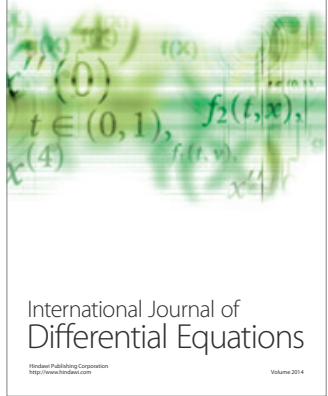
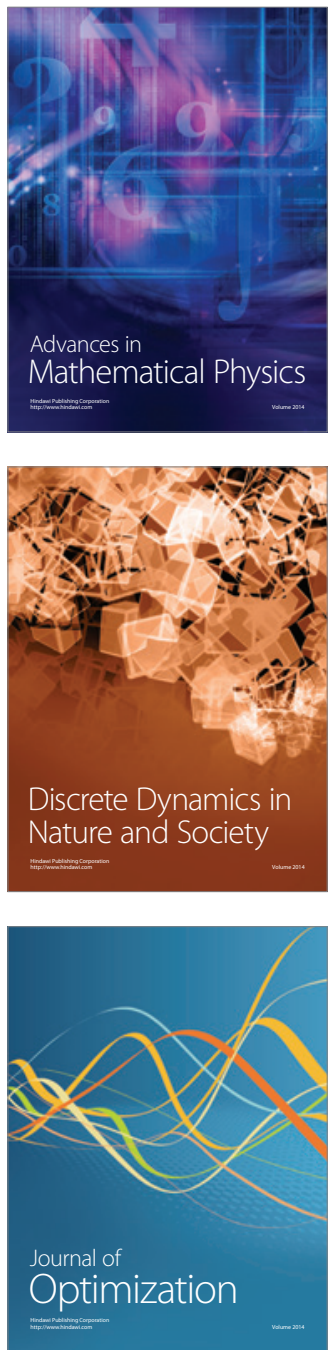